\title{
Clinical Characteristics and Outcome of Primary Sjogren's Syndrome: A Large Asian Indian Cohort
}

\author{
Pulukool Sandhya ${ }^{1}$, Lakshmanan Jeyaseelan ${ }^{2}$, Robert Hal Scofield ${ }^{3}$ and Debashish Danda ${ }^{* 1}$
}

\author{
${ }^{I}$ Department of Clinical Immunology and Rheumatology, Christian Medical College and Hospital, Vellore-632004, \\ Tamil Nadu, India \\ ${ }^{2}$ Department of Biostatistics, Christian Medical College and Hospital, Vellore-632004, Tamil Nadu, India \\ ${ }^{3}$ Arthritis \& Clinical Immunology Program, Oklahoma Medical Research Foundation, Endocrinology, Metabolism and \\ Diabetes, Department of Medicine, University of Oklahoma Health Sciences Center and Department of Veterans Affairs \\ Medical Center, Oklahoma City, OK, USA
}

\begin{abstract}
Objective: To characterise the clinical features, immunological profile and outcome in a cohort of Asian Indian patients with primary Sjögren's syndrome (SS).

Methods: Electronic medical records from a tertiary care teaching hospital in south India were screened for SS between 2004 and 2011. Patients fulfilling American European Consensus group (AECG) 2002 or American College of Rheumatology (ACR) 2012 classification criteria were included. Agglomerative hierarchical cluster analysis to identify patterns of associations between clinical and immunological features was done. Multivariate logistic regression to identify predictors of major systemic involvement was performed. Data on treatment and outcome were retrieved from electronic records.

Results: Of 423 patients suspected to have SS, 332 fulfilled inclusion criteria. Only $8.3 \%$ of patients complained of sicca symptoms on their own at initial presentation. Younger age of onset, higher female to male ratio, paucity of cryoglobulinemia, Raynaud's phenomenon and hyperglobulinemia were unique to this cohort. Cluster analysis revealed two subsets: The first cluster comprised of patients having a major systemic illness with high antibody titers and the second comprised of seronegative patients with mild disease. Over a third of SS cases had severe systemic manifestations necessitating treatment with immunosuppressants. In multivariate logistic regression analysis, anti-Ro and anti-La antibody positivity was associated with higher odds for systemic disease features $(\mathrm{OR}=2.67, \mathrm{P}=0.03$ and $\mathrm{OR}=3.25$, $\mathrm{P}=0.003$, respectively) whereas chronic pain was associated with lower odds $(\mathrm{OR}=0.4, \mathrm{p}=0.032)$. Clinical improvement including symptomatic benefit in sicca and musculoskeletal features was noted with immunomodulators in the majority.

Conclusion: Our cohort of patients with SS has characteristic clinical features; some of them are in contrast with previous observations reported in European patients. This cohort consisted of two distinct patient clusters. The first cluster was associated with major systemic illness and high antibody titers, where as the second cluster comprised of seronegative patients with mild disease. Association of antibody positivity with systemic features was further confirmed on logistic regression analysis.
\end{abstract}

Keywords: Antibodies, cluster analysis, immunosuppressive agents, India, logistic regression analysis, Sjögren’s syndrome.

\section{INTRODUCTION}

Sjögren's syndrome (SS) is an autoimmune disease characterised by lymphocytic infiltration of lacrimal and salivary glands resulting in sicca symptoms. SS has diverse manifestations ranging from mild glandular manifestations to severe extra glandular disease including vasculitis, neuropathy and glomerulonephritis. The disease is poorly studied and is reported to have a varied prevalence between 0.1 and $4.8 \%$ in various populations with an estimated female to male ratio of 9:1 [1]. The diagnosis of SS is usually challenging in clinical settings due to non-specific

*Address correspondence to this author at the Department of Clinical Immunology and Rheumatology, Christian Medical College and Hospital, Vellore-632004, India; Tel: 919442282150; Fax: 91-416-2232035;

E-mail: debashisdandacmc@hotmail.com symptoms at disease onset and presentations of varied disease spectra. These characteristics would mean that a large number of patients remain undiagnosed. In this context, an study describing clinical characteristics and epidemiology of SS is of prime importance in this context.

There are at least 12 criteria till date to classify SS, thereby significantly limiting the possibility to compare data from published literature [2]. Of the criteria available for the diagnosis and evaluation of SS, American European Consensus group (AECG) classification criteria is the most popular [3]. However, certain items in AECG criteria are not routinely used in clinical practice such as salivary flow and sialography. Recently, American College of Rheumatology (ACR) 2012 classification criteria have been proposed [4]. These criteria enable one to make a diagnosis of SS in a patient with suggestive clinical presentation and objective 
evidence such as histopathology and antibodies, even if patient does not volunteer sicca symptoms. A large number of studies on SS have come out in published literature mainly from the European populations, which primarily serve as the source of guiding principles for clinical diagnosis [5-8]. With the exception of a Chinese study, there is a paucity of data on clinical characteristics and epidemiological information from populations other than those of European ancestry [9]. Any comparison of the clinical characteristics of patients studied in these large cohorts is also challenging as each of these studies has a different approach to evaluation and has utilized varied classification criteria.

Interestingly, a number of specific clinical manifestations have been reported from non-European populations. For instance, high frequency of renal tubular acidosis (RTA) in SS has been reported in Chinese and Indian patients [9, 10]. Oriental patients with SS have also been reported to have a higher incidence of protein losing enteropathy [11]. These differences could be at least partially attributed to differences in genetic makeup between the populations $[12,13]$.

It has been suggested that $\mathrm{SS}$ is a rarity in the Indian subcontinent [14]. Earlier studies include a retrospective analysis of 36 patients over a 4 and a half years period from South India published in 2003 and a prospective data of 26 North Indian patients over a 10 year period [14, 15]. In our routine clinical practice at our large tertiary care, teaching hospital, we have realised that most patients with SS may not complain of the classical symptoms even though objective evidence for SS may actually be present. Many of these are patients referred from other specialities with autoantibody positivity and related manifestations suggestive of SS such as low-grade inflammatory arthritis, renal tubular acidosis, chronic pain syndrome, unexplained neuropathy or rarely multiple sclerosis. Hence, these patients are subjected to objective evaluation for SS even in the absence of sicca symptoms, as long as they do not fulfill criteria for any other connective tissue disease. It is likely that SS in the Indian subcontinent may not be as rare a disease as previously thought, and under reporting and under diagnosis of SS may be due to emphasis on sicca symptoms as starting point to diagnose this disease with protean manifestations [14]. We think it is important to create awareness regarding this common autoimmune disease; most medical practitioners including rheumatologists in India as well as developed nations consider this as a rare problem. We wanted to remove this misconception from their minds and also wanted to explain, in a data driven manner, why people miss this diagnosis. By providing data from our cohort of patients with SS, we have tried to highlight the varied facets of the disease.

\section{METHODS}

Institutional Review Board of Christian Medical College, Vellore, India, approved the retrospective study and the study followed the guidelines laid by the committee. Electronic medical records were screened for patients with suspected SS seen at Christian Medical College, Vellore between 2004 and 2011. SS was suspected in patients with non-deforming, Jaccoud type or non-erosive chronic inflammatory arthropathy, polyarthralgia, chronic widespread pain with subtle clues like extensive dental caries, premature loss of teeth, recurrent gingivitis or any systemic feature typical of SS. A SS suspect fulfilling 4 out of 6 AECG criteria was classified as SS [3]. Fulfillment of histopathology (focus score of 1 or above/4 mm square) and serology (combination of positive RF and ANA above 1:320 titer or positive anti-Ro or positive anti-La antibody) as 2 out of 3 criteria defined by ACR 2012 were applied to strong suspects of SS not fulfilling the AECG criteria [4]. Those not fulfilling either of these criteria were excluded from the analysis, although they were under close follow up. Screening for HIV, hepatitis B and hepatitis C was done in all patients and only those with negative serology for these viral agents were included. Patients of SS overlapping with any other well-defined connective tissue disease were excluded too. Histopathology findings on minor salivary gland biopsy from lower lip were noted. Chisholm score greater than 2 was suggestive of SS [16]. Objective evidence for ocular involvement was performed by Schirmer's test. Commercial ELISA kits (Euroimmun, Lubeck, Germany) were used for detection of anti- Ro (or anti-SSA) and anti$\mathrm{La}$ (or anti-SS-B) antibodies and a value of $>20 \mathrm{Ru} / \mathrm{ml}$ was considered to be positive. Unstimulated whole salivary flow, parotid sialography, salivary scintigraphy and ocular staining score were not performed in any of these patients. Outpatient and inpatient records of the patients fulfilling inclusion and exclusion criteria were retrieved. Clinical presentations including extra glandular features, demographic features including disease duration, immunological profile and other lab parameters such as counts, biochemical analyses including thyroid hormones and 25-hydroxyvitamin D3 $(25(\mathrm{OH}) \mathrm{D} 3)$ levels were noted. Levels of 25(OH)D3 lower than $45 \mathrm{nmol} / \mathrm{L}$ by chemiluminescence immunoassay (Roche) were defined as low.

Major systemic involvement was defined as vasculitis or significant involvement of neurological, renal, respiratory tract or haematological systems requiring active interventions including immunosuppression. Features such as isolated leucopoenia, urticaria, trigeminal neuropathy, irritable bowel syndrome and minor extra glandular disease features such as minor musculoskeletal features including arthralgia, chronic non-deforming non-erosive polyarthritis, chronic widespread pain, psychiatric symptoms, bladder symptoms, minor audio vestibular and dental problems were not considered as major systemic manifestations.

Categorical data were expressed as percentage and continuous data were expressed as mean (SD). All statistical analyses were performed with SPSS 16.0 software. A $p$ value of $<0.05$ was considered statistically significant. Agglomerative hierarchical cluster analysis was done to identify patterns of associations between different clinical and immunological features of SS [17, 18]. Phi correlation was calculated as a measure of correlation between the categorical variables. Distance between phi correlation coefficient was calculated using the Euclidian distance and the average linkage method was used to agglomerate the clusters [17, 18]. Multivariate logistic regression analysis was done to identify risk factors for major systemic involvement and seropositivity. Clinically relevant variables with a $\mathrm{p}$ value $<0.10$ in univariate analyses were included in multivariate analysis. We assessed the goodness of fit of the model by Hosmer-Lemeshow chi-square statistics [19]. Odds 
ratio with $95 \% \mathrm{CI}$ and $\mathrm{p}$ values are presented. In addition, the likelihood ratio of anti-Ro and anti-La titers for different systemic manifestations of SS was also computed.

We assessed clinical outcome of patients in terms of joint symptoms, sicca symptoms and systemic involvement at the last recorded follow up visit. Outcome was categorised as absence of symptoms, ongoing improvement, persistence or worsening of symptoms with treatment as reported by the patient or as per physical signs noted by the physician. Patient's global improvement was expressed in percentage as compared to baseline. Data on treatment related outcome were analysed only for patients whose follow up details were available.

\section{RESULTS}

There were 423 patients who were clinically suspected to have SS during the study period. Of these, 294 patients fulfilled AECG classification criteria for SS. SICCA criteria were applied on the remaining 129 patients, and classified an additional 38 patients as SS. Our cohort, therefore, consisted of 332 patients ( 294 by AECG +38 by SICCA criteria); remaining $91 \mathrm{SS}$ suspects who did not fulfill either of the two criteria were excluded from further analysis. They are, however, under close follow up as probable "incomplete Sjögren's".

Of the 332 patients, $315(94.88 \%)$ were women and 17 were men with a female: male ratio of $18.5: 1$. At the time of diagnosis, the mean age (SD) of our cohort was 44.5(10.6) years and the mean disease duration (SD) was 62.65 (60.67) months. The most common subjective presenting feature at first visit was arthralgia or chronic non-deforming arthritis, which was seen in $204(61.4 \%)$ patients; this figure rose to $82.8 \%$ on interrogation and evaluation during follow up. Similarly, only $8.1 \%$ of this cohort voluntarily complained of dry eye or dry mouth at first presentation; although history and clinical evaluation revealed sicca symptoms in large majority (dry mouth $94.3 \%$ and dry eye $88.9 \%$ ). The other initial presenting features were: chronic pain syndrome in 21 patients $(6.3 \%)$, hypokalemic paralysis in $18(5.4 \%)$, glandular swelling in $11(3.3 \%)$, paraesthesia in $8(2.4 \%)$, skin rashes in $7(2.1 \%)$ and nonspecific features in $36(11 \%)$ patients which included thrombocytopenia, liver disease, loss of weight, gangrene (due to vasculitis), cough, fever and renal manifestations.

Glandular, extra glandular and serological features during disease course are shown in Table 1. Notably, 121 patients $(36.45 \%)$ had major systemic involvement with considerable overlaps, as some patients had 1 or more such manifestations involving 1 or more systems.

Renal manifestations were seen in $47(14.16 \%)$ patients. This included 10 patients with chronic interstitial nephritis and 7 with glomerulonephritis. Renal biopsy was not done in 14 patients with subnephrotic proteinuria as the patients did not agree to undergo the procedure. RTA was seen in 27 (8.1\%) patients. Four patients had renal stones, 2 had medullary nephrocalcinosis, and 1 patient had both renal stones and medullary nephrocalcinosis. One patient each also had chronic kidney disease and medullary cystic kidney disease. Urological manifestations included polyuria or increased urinary frequency in 17 , hydroureteronephrosis in
3 , urethral stricture in 1 and recurrent urinary tract infection in 1 patient.

Of 40 patients with respiratory involvement, 21 had interstitial lung disease documented by high resolution computed tomography (HRCT) and spirometry, 10 had obstructive lung disease, 4 had bronchiectasis identified by HRCT and 2 had pleural effusion. One patient each had alveolar haemorrhage, pulmonary thromboembolism and pulmonary nodules.

Table 1. Features of 332 patients with SS during disease course.

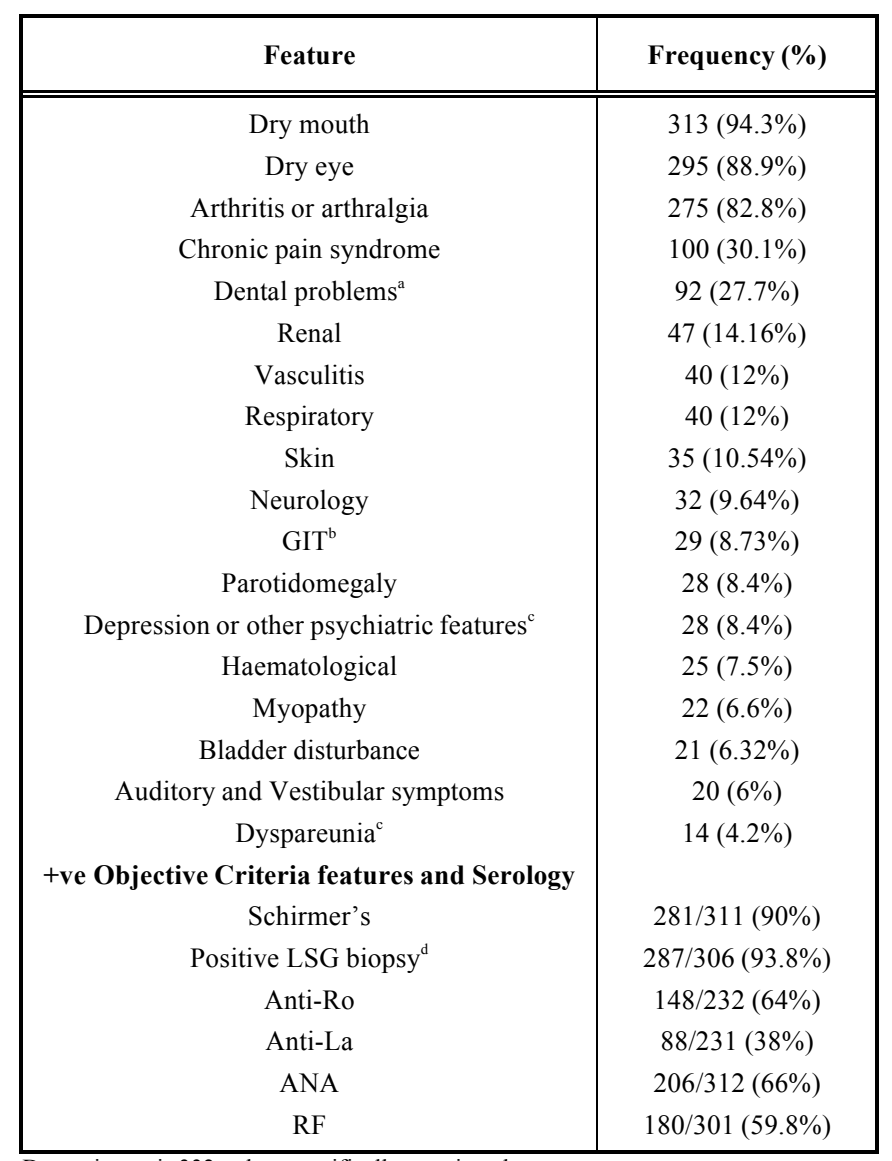

Denominator is 332 unless specifically mentioned.

${ }^{a}$ Dental features - as documented by dentist or physician.

${ }^{\mathrm{b}} \mathrm{GIT}$ - Gastrointestinal tract.

${ }^{\mathrm{c}}$ Depression/Psychiatric symptoms and dyspareunia as documented by psychiatrist and gynaecologist, respectively.

${ }^{\mathrm{d}}$ LSG- Labial salivary gland.

Among those with skin manifestations, the following features were noted (number within bracket denotes number of patients): Palpable purpura(14), erythematous rash(8), urticaria(7), photosensitivity(6), erythema nodosum(2), Raynaud's phenomenon [RP] (1), atrophie blanche(1) and gangrene(1).

Neurological manifestations seen in our cohort were as follows: Mononeuritis multiplex (20), symmetrical polyneuropathy (5), carpal tunnel syndrome (4), trigeminal neuropathy (1), recurrent seizures \& aseptic meningitis (1), chronic immune demyelinating polyneuropathy (1) and dysautonomia (1). Of the 22 patients with myopathy, all except one patient had proximal myopathy secondary to RTA. One patient had associated biopsy proven 
inflammatory myositis. Two patients had vestibular dysfunction and 18 had sensorineural hearing loss.

A number of patients also had haematological manifestations. Break down of haematological manifestations was as follows: thrombocytopenia in 14, leucopenia in 2, pseudolymphoma in 2, monoclonal gammopathy of undetermined significance in 4, Kikuchi's disease in 1 and unexplained hepatosplenomegaly in 2 patients.

Infertility was seen in 4 women: 2 had primary and the remaining two had secondary infertility. Two patients had 1 fetal death each resulting from complete heart block. Other than this, multiple $(\geq 2)$ spontaneous abortions were documented in 14 patients, of whom 8 were positive for antiRo and anti-La antibody, 3 were positive for anti-Ro antibody alone, 2 patients had antiphospholipid antibody syndrome and 1 patient was not subjected to these autoantibody tests. None of these 14 patients had documented fetal heart block as per hospital records.

Mean (SD) titers of RF, anti-Ro and anti-La antibodies were 226.2 (208.8) kIU/L, 165.3 (57.7) $\mathrm{Ru} / \mathrm{ml}$ and 173.7 (92.3) $\mathrm{Ru} / \mathrm{ml}$ respectively. Hyperglobulinemia was seen in only 50 patients out of $261(15.4 \%)$ in whom it was looked for. Cryoglobulinemia was not detected in any of the patients. 25(OH)D3 levels were available for 236 patients and the mean (SD) was 47.33(28) $\mathrm{nmol} / \mathrm{L}$. Hypothyroidism was seen in 102 out of 308 patients tested (33.11\%). Only one patient had Graves' disease.

Cluster analysis results of clinical and immunological characteristics as described earlier are depicted in Fig. (1). The cluster analysis indicated that our cohort consisted of two subsets of SS population. I - The first subset included those with serological positivity (ANA, RF, anti-Ro, anti-La) and extra glandular major systemic manifestations namely dermatological, vasculitic, neurological and renal involvement requiring high dose steroids. II - The other subset consisted of those with sicca symptoms, low $25(\mathrm{OH})$ D3 $(<45 \mathrm{nmol} / \mathrm{L})$ and generally mild manifestations including chronic pain, arthralgia or non-deforming chronic arthritis etc. This subset did not cluster with serological positivity.

Results of multivariate logistic regression for identifying predictors of major systemic involvement and associations of anti-Ro and anti-La positivity are depicted in Table 2. Major systemic features were positively associated with anti-Ro and anti-La antibody and negatively with chronic pain. Logistic regression for association of anti-Ro and anti-La

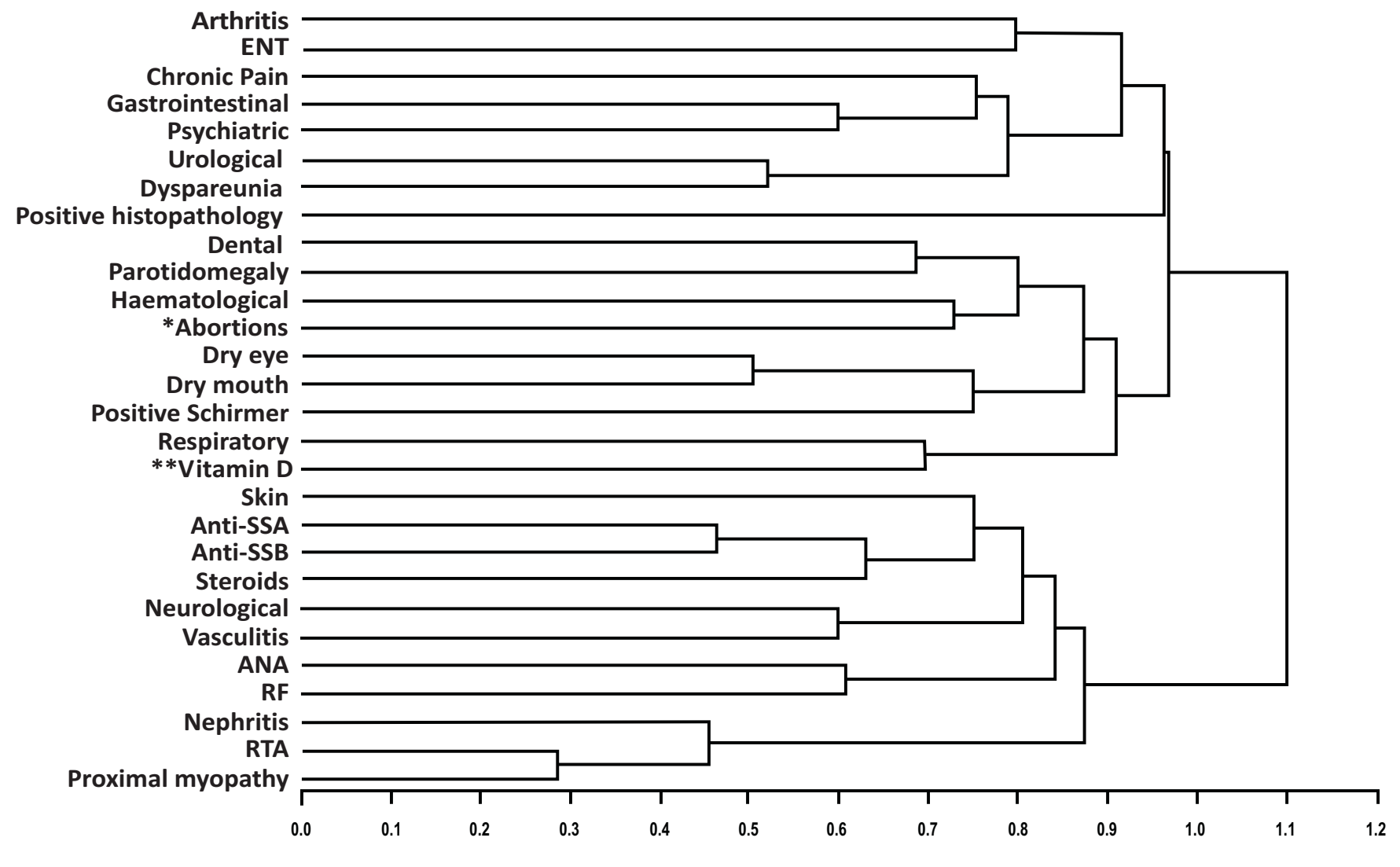

AVERAGE DISTANCE BETWEEN CLUSTERS

*Abortions implies two or more spontaneous abortions

**Vitamin D -25-hydroxy vitamin D $<45 \mathrm{nmol} / \mathrm{L}$

Fig. (1). Cluster analysis of clinical and immunological characteristics. Agglomerative hierarchical cluster analysis was done to identify patterns of associations between different clinical and immunological features of SS. Phi correlation was calculated as a measure of correlation between the categorical variables. Distance between phi correlation coefficient was calculated using the Euclidian distance and the average linkage method was used to agglomerate the clusters. 
Table 2. Adjusted Logistic regression of major systemic involvement, anti-Ro and anti-La seropositivity.

\begin{tabular}{|c|c|c|c|}
\hline Parameter & Odds Ratio & 95\% C.I. & P-Value \\
\hline \multicolumn{4}{|c|}{ Predictors of major systemic involvement } \\
\hline Anti-Ro & 2.67 & $1.09-6.54$ & 0.03 \\
\hline Anti-La & 3.25 & $1.49-6.9$ & 0.003 \\
\hline $25(\mathrm{OH}) \mathrm{D}_{3}$ & 0.63 & $0.31-1.29$ & 0.2 \\
\hline ARTHRITIS & 0.47 & $0.2-1.14$ & 0.1 \\
\hline $\mathrm{CPS}^{\text {a }}$ & 0.4 & $0.17-0.93$ & 0.032 \\
\hline \multicolumn{4}{|c|}{ Associations of anti-Ro and anti-La antibodies } \\
\hline ARTHRITIS & 0.39 & $0.19-0.81$ & 0.01 \\
\hline $\mathrm{CPS}^{\mathrm{a}}$ & 0.47 & $0.21-1.04$ & 0.06 \\
\hline SKIN & 3.9 & $1.57-9.7$ & 0.003 \\
\hline NEPHRITIS & 1.84 & $0.74-4.6$ & 0.19 \\
\hline $\mathrm{RTA}^{\mathrm{b}}$ & 2.61 & $0.77-8.84$ & 0.12 \\
\hline NEUROLOGICAL & 1.31 & $0.45-3.83$ & 0.63 \\
\hline MYOPATHY & 2.19 & $0.57-8.4$ & 0.25 \\
\hline VASCULITIS & 1.47 & $0.54-4.0$ & 0.454 \\
\hline ANA & 4.99 & $2.08-11.98$ & 0.000 \\
\hline
\end{tabular}

${ }^{\mathrm{a} C P S}$ - Chronic Pain Syndrome.

${ }^{\mathrm{b}} \mathrm{RTA}$ - Renal Tubular Acidosis.

antibody revealed significantly higher odds ratio for skin manifestations and ANA positivity. Presence of arthritis, however, had significant negative association with anti-Ro and anti-La antibody as depicted in Table 2.

Logistic regression analysis of serological positivity and systemic features revealed that anti-Ro and anti-La positivity had a positive correlation with the systemic features. In addition, likelihood ratio calculation revealed that patients with systemic features had a 2 to 3 times higher odds to have high antibody titers $(>160 \mathrm{Ru} / \mathrm{ml})$ of anti-Ro and anti-La antibodies.

\section{Treatment and Follow Up}

Follow up data were available for 229 patients. The median duration of follow up was 24 (3-192) months.

Glucocorticoids. Seventy-eight patients were on low dose glucocorticoids ( $\leq$ prednisolone $10 \mathrm{mg}$ or equivalent) at some point of time during the course of follow up. Fifty patients received high dose glucocorticoids for severe systemic manifestations that included 26 with vasculitis, 8 with interstitial lung disease, 5 with haematological manifestations, 4 each with autoimmune hepatitis or glomerulonephritis, and 1 each with chronic inflammatory demyelinating polyneuropathy or alveolar haemorrhage, respectively. Some of these patients required added immunosuppression with mycophenolate mofetil, azathioprine, cyclophosphamide or methotrexate.

Immunomodulators/Immunosuppressants. Details of medications including immunomodulators and immunosuppressants in 229 patients on follow up are depicted in Table 3.
At the last follow up visit, 214 patients (93.5\%) were on hydroxychloroquine either, alone or in combination with other agents. Methotrexate, alone or in combination, was the second most common medication used by 120 patients

Table 3. Medications prescribed in SS cohort.

\begin{tabular}{|c|c|}
\hline Immunomodulators and Immunosuppressants & $\mathbf{N = 2 2 9}$ \\
\hline \hline HCQ & 49 \\
\hline HCQ + MTX & 90 \\
\hline HCQ + Omega Fatty Acid & 20 \\
\hline HCQ + MMF & 24 \\
\hline HCQ + MTX + SSZ & 14 \\
\hline HCQ + MTX + Omega Fatty Acid & 10 \\
\hline HCQ + AZA & 7 \\
\hline MTX & 5 \\
\hline AZA & 5 \\
\hline MMF & 1 \\
\hline CYC & 1 \\
\hline MTX + SSZ & \\
\hline
\end{tabular}

HCQ - Hydroxychloroquine.

MTX- Methotrexate.

AZA- Azathioprine.

SSZ- Sulphasalazine.

MMF- Mycophenolate Mofetil.

CYC- Cyclophosphamide.

$(52.4 \%)$. Ninety patients $(39.3 \%)$ were on combination of hydroxychloroquine and methotrexate. Thirty patients were 
on omega-3 fatty acids (Eicosapentanoic acid and Docosahexanoic acid) in combination with hydroxychloroquine and/or methotrexate. Thirteen patients were temporarily on sulphasalazine in combination with methotrexate and/or hydroxychloroquine, as they were initially suspected to have rheumatoid arthritis (RA). None of these patients had deforming arthritis or erosions on x-rays; hence, sulphasalazine was discontinued eventually once the diagnosis of SS was established. Sulphasalazine was, however, added in 3 patients on methotrexate and hydroxychloroquine combination and in 1 patient on methotrexate alone in view of persistent synovitis. Overall, drug therapy was escalated in 11 patients including use of rituximab in 1 patient with major systemic involvement during follow up. Downgrading of therapy was done in 20 patients after sustained control of disease.

\section{Outcome}

At follow up, physician documented outcome was available for 161 out of $275(58.6 \%)$ patients with baseline joint symptoms. Similar data were available for 157 out of $313(50.16 \%)$ patients and 72 out of $121(59.5 \%)$ patients with sicca symptoms and systemic involvement, respectively (Fig. 2).

Outcome of joint symptoms. Break down of 161 patients with available outcome data was as follows: Asymptomatic on treatment $-131(81.4 \%)$, persistence $-11(6.8 \%)$, ongoing improvement $-19(11.8 \%)$. Worsening of joint symptoms was not seen in any patient at the last documented follow up. Of the 131 patients who were asymptomatic in terms of joint symptoms on treatment, 127 were on methotrexate, hydroxychloroquine or both agents. Additional omega-3 fatty acids or sulphasalazine were given to 19 and 4 of these patients, respectively. Mycophenolate mofetil, azathioprine or cyclophosphamide was added to 18 patients for associated major systemic features; ongoing methotrexate therapy in these 18 patients was stopped. Mycophenolate mofetil or glucocorticoid alone as single agent was used in 1 patient each. Only 2 out of these 161 patients with musculoskeletal symptoms were not on any medication.
Outcome of sicca symptoms. Break down of 157 patients is as follows: asymptomatic on treatment $-55(35 \%)$, ongoing improvement -74(47\%), persistence -28(18\%). Again, no patient reported worsening of symptoms. Outcome of sicca symptoms was also assessed by documented decrease in use of tear and saliva supplements, apart from symptoms reported by the patients. None of these patients with sicca symptoms were on secretagogues.

Of the 55 patients who were asymptomatic on treatment, 51 were on hydroxychloroquine, methotrexate or both. Omega-3 fatty acid, sulphasalazine, mycophenolate mofetil or azathioprine was added to 4, 4, 6 and 3 patients, respectively. One patient was on glucocorticoids alone and 2 patients were off all drugs. Of the 74 patients with improvement in sicca symptoms, 73 were on hydroxychloroquine, methotrexate or both. Omega3 fatty acid, mycophenolate mofetil, azathioprine or cyclophosphamide was prescribed to $9,8,6$ and 2 patients, respectively. One patient was on omega3 fatty acid alone.

Outcome with regards to systemic involvement. Of 72 patients with available follow up data, 64 (89\%) were asymptomatic on immunosupressants. Ongoing improvement was noted in $6(8 \%)$, whereas worsening was seen in only 2 (3\%) patients. Of the 64 patients asymptomatic on treatment, use of steroid sparing immunosuppressants was as follows: mycophenolate mofetil (19), azathioprine (6), cyclophosphamide (4) and rituximab (1). Hydroxychloroquine, methotrexate or both agents were also prescribed to 59 of these patients. There was, however, no concurrent use of methotrexate with mycophenolate mofetil, azathioprine or cyclophosphamide.

Global improvement. Patient reported global improvement was available for 146 out of 229 patients who came for follow up. Median global improvement at last follow up visit as compared to baseline was 70\% (10-100 scale). Presence of arthralgia, fibromyalgia, glandular swelling, systemic involvement, positive lip biopsy or serology did not predict patient reported global improvement in logistic regression analysis.

Incidence of malignancy. Of 423 patients (332 with definite SS fulfilling either or both criteria and 91 strong suspects of SS

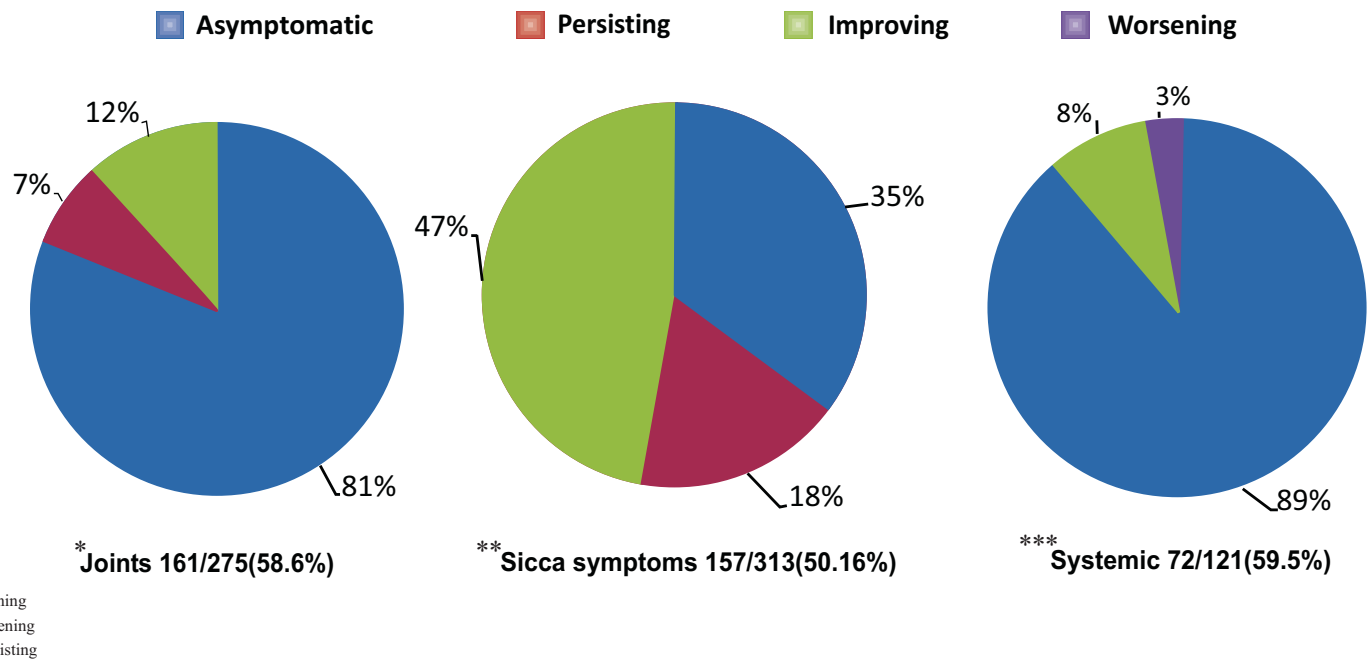

Fig. (2). Pie-chart depicting outcome of treatment in terms of joint symptoms, sicca symptoms and systemic involvement. 
who did not fulfill either classification criteria), 1 patient each was diagnosed to have B cell lymphoma, parotid lymphoma and acute myeloid leukemia based on histopathology. Two other strong suspects for malignancy refused biopsy. One amongst these 2 patients had small vessel cutaneous vasculitis and multiple enlarged abdominal nodes. Fine needle aspiration cytology in this patient was suggestive of lymphoma. The other had ascites and raised alpha-fetoprotein in the background of associated autoimmune hepatitis and was suspected to have hepatocellular carcinoma or lymphoma. There was no mortality as per the hospital records in this cohort.

\section{DISCUSSION}

This cohort of patients with SS is the first large series from the Indian subcontinent. We have compared this cohort with 5 other large cohorts [5-9] in Table 4. Almost all of the earlier studies except one were based on European populations. Apart from the present study, the only other large study from Asia was from China in 2010 [9].

One of the significant findings from this study was the observation that at the time of diagnosis, our patients were younger by a decade as compared to the cohorts of European ancestry. This was also observed in the Chinese population [9]. In addition, the female: male ratio was higher than most published series. Integral features of SS like sicca symptoms were subjective presenting complaints only in $8.1 \%$ of our patients, even though interrogation and objective evaluation could detect the same in most of them. A similar observation was also made in the previous Chinese series where only a small proportion of the patients presented with sicca symptoms [9]. This could imply subclinical or mild

Table 4. Comparison of our series with other major large series of Sjögren's syndrome.

\begin{tabular}{|c|c|c|c|c|c|c|}
\hline Parameter & $\begin{array}{l}\text { Finnish } \\
1977-92\end{array}$ & $\begin{array}{c}\text { Spain } \\
\text { 1994-2007 }\end{array}$ & $\begin{array}{c}\text { French } \\
\text { 1985-2009 }\end{array}$ & $\begin{array}{c}\text { Swedish } \\
\text { 1984-2004 }\end{array}$ & $\begin{array}{c}\text { Chinese } \\
\text { 1985-2006 }\end{array}$ & $\begin{array}{c}\text { Present Study } \\
\text { 2004-2011 }\end{array}$ \\
\hline Number of Patients & 110 & 1010 & 445 & 205 & 573 & $332^{9}$ \\
\hline Classification Criteria Used & $\begin{array}{l}\text { Californian/ } \\
\text { European } \\
\text { Community }\end{array}$ & $\begin{array}{c}\text { European } \\
\text { Community }\end{array}$ & AECG & AECG & AECG & $\begin{array}{c}\text { AECG } \\
\text { ACR }\end{array}$ \\
\hline Number of Centres & 1 & 10 & 2 & 1 & 1 & 1 \\
\hline Mean age (yrs) & $62 \pm 13^{a}$ & $53.0 \pm 0.48$ & $53.6 \pm 14$ & & 39 & $44.5 \pm 10.6$ \\
\hline Disease duration(mo) & & $74.9 \pm 4$ & & & & $62.65 \pm 65.67$ \\
\hline Female:Male & $35.7: 1$ & $13: 1$ & $8.9: 1$ & & $10.7: 1$ & $18.5: 1$ \\
\hline Arthralgia/arthritis & $106(97)$ & $640(63)$ & $222(50)$ & (76) & $274(47.8)$ & $275(82.8)$ \\
\hline Raynaud's & $55(50)$ & $187(18)$ & $189(42)$ & (20) & $101(17.6)$ & $1(0.3)$ \\
\hline Pulmonary & $33(31)^{b}$ & $112(11)$ & $55(12)$ & $38(19)$ & $221 / 522(42.3)$ & $40(12.1)$ \\
\hline Renal & & $48(5)$ & $34(8)$ & $78(38)$ & $192(33.5)$ & $49(14.75)$ \\
\hline GI/hepatobiliary & & $5(0.5)^{\mathrm{c}}$ & & $26(12)^{d}$ & $188(32.8)$ & $29(8.73)$ \\
\hline Neurological & $35(32)$ & $131(13)$ & $70(16)$ & & $68(11.9)$ & $32(9.64)$ \\
\hline Vasculitis & & $91(9)$ & $70(16)$ & & & $40(12)$ \\
\hline Positive LSG biopsy & $77(70)$ & $449 / 561(79)$ & $403(92 \%)$ & & $303 / 334(90.7)$ & $287 / 306(93.8)$ \\
\hline Subjective oral sicca & $97(88)$ & $975(96)$ & $380(86)^{\mathrm{e}}$ & & $484(84.5)$ & $313 / 332(94.3)$ \\
\hline Subjective ophthalmic sicca & $86(72)$ & $968(96)$ & $371(83.4)^{\mathrm{e}}$ & & $401(70)$ & $295 / 332(88.9)$ \\
\hline ANA & $(83)^{g}$ & $859 / 1005(85)^{\mathrm{f}}$ & $347(78)^{\mathrm{g}}$ & $101 / 170(59)$ & $474 / 565(83.9)$ & $206 / 312(66)^{g}$ \\
\hline RF & $(74)$ & $467 / 982(48)$ & $184(41)$ & & $292 / 476(61.3)$ & $180 / 301(59.8)$ \\
\hline Anti-Ro & & $518 / 1002(52)$ & $221(48)$ & $(61)^{\mathrm{h}}$ & $474 / 568(83.5)$ & $148 / 232(63.8)$ \\
\hline Anti-La & & $343 / 1000(34)$ & $139(31)$ & $(41.5)^{\mathrm{h}}$ & $231 / 568(40.7)$ & $88 / 231(38.1)$ \\
\hline Cryoglobulinemia & & $62 / 628(10)$ & $68(15)$ & & & $0 / 69$ \\
\hline Hyperglobulinemia & & & $225(50)$ & & & $50 / 261(15.4)$ \\
\hline Follow up (months) & $108(1.2-216)$ & & $76.1 \pm 51$ & & & $31 \pm 29$ \\
\hline
\end{tabular}

Continuous data are expressed as mean $\pm \mathrm{sd}$; Categorical data are expressed as absolute frequency $\mathrm{n}(\%)$.

NA Data not available.

AECG American-European consensus group criteria.

ACR American College of Rheumatology.

${ }^{a}$ only age at last visit given.

bulmonary fibrosis and pleurisy.

includes only pancreatitis.

dinvolvement of liver and pancreas.

${ }^{\mathrm{e}}$ at time of diagnosis.

${ }^{\mathrm{f}}$ IIF(triple tissue liver-stomach-kidney)

${ }^{\mathrm{g}} \mathrm{Hep}-2$.

${ }^{\mathrm{h}}$ In house ELISA 
exocrinopathy in early disease or potential population level differences, which could be due to differences in genetic make up between Asian and Caucasian populations [12, 13]. The high prevalence of musculoskeletal symptoms in this Indian cohort is comparable to most published series as shown in Table 4. One-third of our patients had chronic pain features, which is also a well described manifestation of SS adversely affecting quality of life in these patients [20,21].

This study also demonstrates that a proactive evaluation for SS in rheumatology clinic has a high yield. Presence of subtle features like sequelae of oral and ocular sicca or any known systemic manifestation of SS could be pointers with high pretest probability for the diagnosis of SS. Awareness and familiarity with these clues should prompt the rheumatologist to suspect SS in the setting of mild musculoskeletal features like arthralgia, chronic nondeforming inflammatory polyarthritis and chronic pain. In our cohort of patients, the SICCA criteria, even without the ocular staining score, enabled us to diagnose 38 patients in addition to those diagnosed by AECG criteria alone. This is because many of these patients had both ANA (in titer of 1:320 and above) and RF positivity, which is included as a serological criterion exclusive of anti-Ro and anti-La antibody in SICCA criteria, but not in AECG criteria. In the absence of subjective symptoms, AECG criteria requires 3 out of 4 objective criteria to be fulfilled, whereas SICCA criteria needs only 2 out of 3 objective criteria for diagnosis of SS. ANA and RF testing are more frequently ordered by general physicians and other medical practitioners prior to referral to Rheumatology department. This may be an advantage for rheumatologists, as a referred patient with both positive RF and ANA in 1:320 titer, therefore, needs just a positive salivary gland biopsy to be diagnosed as SS by SICCA criteria. Evaluation for objective oral signs of salivary flow can be a cumbersome and limiting factor in routine work up for SS by AECG criteria and the same may be true for ocular staining score by SICCA criteria. We, therefore, suspect that we could have missed out a few more SS cases amongst the 91 patients not fulfilling either criteria (of the 423 total suspects), as we did not do objective assessment of salivary flow defined by AECG classification, nor did we do ocular staining score for SICCA criteria. These 91 patients with probable "incomplete Sjögren's" are, however, under close follow up.

RP in only 1 patient and absence of cryoglobulinemia in our cohort is unlikely to be due to warm climate alone. Exclusion of patients overlapping with other connective tissue diseases like systemic sclerosis and mixed connective tissue disease from our series also may not fully explain such low prevalence of RP. This is a distinctly unique feature of our cohort. A higher prevalence of clinically overt RTA was also noted in our series as well as in the Chinese series [9, $10,22,23]$.

We also suspect that some of the glandular and extra glandular features may have been under reported in our series in view of retrospective nature of our study. In addition, patients with SS may present with several protean, non-sicca manifestations to specialities unfamiliar with this illness and its features.

Another interesting finding that has come out of the present study is the observation of two distinct clusters.
These two subsets of SS generated by cluster analysis in the present series contrasts the clusters in the French series in many ways. The subset with significant systemic complications and immunological abnormalities in this Indian series did not show close association with positive LSG biopsy, sicca symptoms, articular manifestations, low vitamin D or chronic pain features. In the French cohort, Martel et al. reported clustering of articular involvement, RP, cutaneous vasculitis, cryoglobulinemia, peripheral neuropathy and renal involvement. Anti-Ro and anti-La positivity, hyperglobulinemia and parotidomegaly formed a separate cluster in their study. No clustering was seen between systemic manifestation and serology or focus score on LSG biopsy in that series [6].

Logistic regression analysis of our data underscores the importance of anti-Ro and anti-La antibodies as predictors of major systemic disease. High titers of antibodies usually reflect B cell hyperactivity leading on to hyperglobulinemia and cryoglobulinemia, known prognostic factors in SS [6, 24]. Whether absence of hyperglobulinemia and cryoglobulinemia in this cohort could denote a better prognosis remains to be seen. There is indeed lower incidence of lymphoma and no mortality in this series. Higher attrition and shorter duration of follow up may explain this phenomenon to some extent [25], or is this another pointer to a milder phenotype of disease or better prognosis in Indian patients due to genetic, epigenetic or dietary factors?

A high positive likelihood ratio for high titer anti-Ro/La antibodies in patients with major systemic features is a novel finding which may suggest a possible pathogenic role of these autoantibodies. Chronic pain, on the other hand, as an independent negative association for major systemic features as revealed by logistic regression analysis has not been reported previously either $[5,6,24,26]$. This analysis also found arthralgia/arthritis to have negative association with anti-Ro and anti-La positivity, thereby, adding further strength to the findings of the cluster analysis.

Evaluation of clinical outcome of this cohort had limitations as it was based on physician assessment and patient reported subjective global improvement, rather than recently introduced objective outcome measures such as EULAR Sjögren's Syndrome Patient Reported Index (ESSPRI) and EULAR Sjögren's Syndrome Disease Activity Index (ESSDAI) [27, 28]. However, favourable outcome in global health, sicca symptoms and musculoskeletal features with immunomodulatory agents like methotrexate and hydroxychloroquin were noted in vast majority of the patients, in addition to predictable benefit in systemic features with immunosuppressants. Contrary to beliefs, several reports of similar improvements in glandular and extra glandular features of SS with immunomodulatory treatment do exist in literature [29-33]. However, our cohort is limited by selection bias, as is the case with most retrospective data from tertiary care referral hospitals. This clinical spectrum hence may not be reflective of the disease in the community.

Omega-3 fatty acids are known to have anti-inflammatory properties by virtue of multiple mechanisms including inhibitory effects on pro-inflammatory cytokines and therefore, they have been found to be useful in the treatment of inflammatory arthritic conditions $[34,35]$. With this biological basis in mind, omega-3 fatty acid has been successfully used as a safe and natural adjunct to therapy amongst consenting 
patients of this SS cohort with minor extra glandular involvement. Secretagogues are not commonly used in Indian patients due to poor availability and cost constraints; moreover, improvement in sicca symptoms with immunomodulatory treatment leading to reduction in use of saliva and tear supplements further obviated the need for such agents. It is also likely that sicca symptoms were probably milder in these patients, as only a small proportion voluntarily complained of sicca symptoms in spite of being elicitable in most of them by their physicians. Mild sicca amongst these patients responding well to low dose methotrexate, hydroxychloroquine or omega-3 fatty acid may, therefore, define another distinctive feature of $\mathrm{SS}$ in Asian Indian patients in contrast to their western counterparts.

Use of immunomodulatory agents like methotrexate and hydroxychloroquine seems biologically logical as immune mediated damage underlies pathogenesis of SS. Moreover, biopsy of labial salivary glands even after many years of disease shows predominantly active inflammatory infiltrate in most cases, rather than gross fibrosis or atrophy [36]. This implies that potentially reversible pathology does exist in SS even late in the disease process. Therefore, there is scope for symptomatic improvement in SS with these mild immunomodulatory agents, as in other systemic autoimmune diseases. Paucity of large clinical trials has kept the literature divided on this issue, rather than any hard evidence. Data from the present cohort, in spite of having a very large number of SS patients, are also limited by its retrospective nature. Generating high level evidence by multicentric, randomised, controlled trials or comparative effectiveness trials for efficacy of these safe, inexpensive agents in SS using objective tools is imperative in this era of global resource crunch.

Finally, several unique features of this cohort namely younger age of onset, higher female: male ratio, higher musculoskeletal symptoms like chronic pain, underplay of sicca symptoms by our patients at presentation, paucity of parotidomegaly, hyperglobulinemia, RP and cryoglobulinemia, distinct clustering of seropositive major systemic versus mild disease, better outcome with treatment and lower incidence of malignancy are distinctive of Indian patients with SS. All of these observations could imply interplay of genetic and ethnic variations under different environmental and epigenetic pressures including yet unknown infective triggers or even dietary modifications in Indian patients with SS. It warrants genomic, transcriptomic and proteomic studies in this research naïve SS patients from India. Alternatively, can some of these favourable variations in our cohort be due to early diagnosis of SS much before advanced glandular and extra glandular damage is established?

\section{CONCLUSION}

This series reiterates the fact that high degree of suspicion and proactive evaluation can pick up SS from patients with low-grade musculoskeletal symptoms in rheumatology clinic, even when patients underplay sicca symptoms. Keeping subtle systemic features of SS and known sequelae of sicca complications in mind as clues with high pretest probability may hold the key for diagnosing SS in such settings. This approach, therefore, may diagnose SS early and make patients amenable to treatment with favourable outcome as in other systemic autoimmune diseases like early RA. In this cohort, seronegative patients had a mild form of disease, while anti-Ro and/or anti-La positivity was associated with major systemic manifestations. Immunomodulatory treatment in the former and immunosuppressive treatment in the latter subset were beneficial in ameliorating glandular and extra glandular features in a high proportion of Asian Indian patients with SS.

\section{KEY MESSAGES}

1. In this large Asian Indian cohort, majority of patients with primary SS did not present with sicca symptoms as the initial presenting complaint, even though it could be elicited in the vast majority of them by interrogation and evaluation.

2. Two distinct subsets of SS identified include: A. Major systemic disease associated with high titer SS-A/SS-B antibody responsive to immunosuppressive treatment and B. Seronegative disease associated with mild features like sicca symptoms and musculoskeletal aches, majority of whom responded to immunomodulatory agents.

3. RP, hyperglobulinemia and cryoglobulinemia are rare in Indian patients of SS.

\begin{tabular}{|c|c|c|}
\hline \multicolumn{3}{|c|}{ ABBREVIATIONS } \\
\hline 25(OH)D3 & $=$ & 25-hydroxyvitamin D3 \\
\hline ACR & $=$ & American College of Rheumatology \\
\hline AECG & $=$ & American-European consensus group \\
\hline ANA & $=$ & Antinuclear antibodies \\
\hline ESSDAI & $=$ & $\begin{array}{l}\text { EULAR Sjögren's Syndrome Disease } \\
\text { Activity Index }\end{array}$ \\
\hline ESSPRI & $=$ & $\begin{array}{l}\text { EULAR Sjögren's Syndrome Patient } \\
\text { Reported Index }\end{array}$ \\
\hline HRCT & $=$ & High resolution computed tomography \\
\hline LSG & $=$ & Labial salivary gland \\
\hline RA & $=$ & Rheumatoid arthritis \\
\hline $\mathrm{RF}$ & $=$ & Rheumatoid Factor \\
\hline $\mathrm{RP}$ & $=$ & Raynaud phenomenon \\
\hline RTA & & Renal tubular acidosis \\
\hline SS & & Sjögren's syndrome \\
\hline
\end{tabular}

\section{CONFLICT OF INTEREST}

The authors confirm that this article content has no conflict of interest.

\section{ACKNOWLEDGEMENTS}

The authors gratefully acknowledge the assistance provided by Ms. Devika, Mr. Silambarasan and Mr. BijeshYadav in statistical analysis. We thank the staff of the Department of Clinical Immunology and Rheumatology and Medical Records Department for their support. 


\section{REFERENCES}

[1] Mavragani CP, Moutsopoulos HM. Thegeoepidemiology of Sjögren's syndrome. Autoimmun Rev 2010; 9: A305-10.

[2] Daniels TE. Do we need new diagnostic criteria for Sjögren's syndrome? Presse Med 2012; 41: e441-9.

[3] Vitali C, Bombardieri S, Jonsson R, et al. European Study Group on Classification Criteria for Sjögren's Syndrome. Classification criteria for Sjögren's syndrome: a revised version of the European criteria proposed by the American-European Consensus Group (review). Ann Rheum Dis 2002; 61: 554-8.

[4] Shiboski SC, Shiboski CH, Criswell L, et al. Sjögren's International Collaborative Clinical Alliance (SICCA) Research Groups. American College of Rheumatology classification criteria for Sjögren's syndrome: a data-driven, expert consensus approach in the Sjögren's International Collaborative Clinical Alliance cohort. Arthritis Care Res 2012; 64: 475-87.

[5] Locht H, Pelck R, Manthorpe R. Clinical manifestations correlated to the prevalence of autoantibodies in a large $(n=321)$ cohort of patients with primary Sjögren's syndrome: a comparison of patients initially diagnosed according to the Copenhagen classification criteria with the American-European consensus criteria. Autoimmun Rev 2005; 4: 27681 .

[6] Martel C, Gondran G, Launay D, et al. Active immunological profile is associated with systemic Sjögren's syndrome. J Clin Immunol 2011; 31: 840-7.

[7] Pertovaara M, Pukkala E, Laippala P, Miettinen A, Pasternack A. A longitudinal cohort study of Finnish patients with primary Sjögren'ssyndrome: clinical, immunological, and epidemiological aspects. Ann Rheum Dis 2001; 60: 467-72.

[8] Ramos-Casals M, Solans R, Rosas J, et al. GEMESS Study Group. Primary Sjögren syndrome in Spain: clinical and immunologic expression in 1010 patients. Medicine (Baltimore) 2008; 87: 210-9.

[9] Lin DF, Yan SM, Zhao Y, et al. Clinical and prognostic characteristics of 573 cases of primary Sjögren's syndrome. Chin Med J (Engl) 2010; 123: 3252-7.

[10] Sandhya P, Danda D, Rajaratnam S, Thomas N. Sjögren's, Renal Tubular Acidosis And Osteomalacia - An Asian Indian Series. Open Rheumatol J 2014; 8: 84-90.

[11] Nagashima $\mathrm{T}$, Hoshino $\mathrm{M}$, Shimoji $\mathrm{S}$, et al. Protein-losing gastroenteropathy associated with primary Sjögren's syndrome: a characteristic oriental variant. Rheumatol Int 2009; 29: 817-20.

[12] Lessard CJ, Li H, Adrianto I, et al. Variants at multiple loci implicated in both innate and adaptive immune responses are associated with Sjögren's syndrome. Nat Genet 2013; 45: 1284-92.

[13] Li Y, Zhang K, Chen H, et al. A genome-wide association study in Han Chinese identifies a susceptibility locus for primary Sjögren's syndrome at 7q11.23. Nat Genet 2013; 45: 1361-5.

[14] Misra R, Hissaria P, Tandon V, Aggarwal A, Krishnani N, Dabadghao S. Primary Sjogren's syndrome: rarity in India. J Assoc Physicians India 2003; 51: 859-62.

[15] Porkodi R, Rukmangatharajan S, Kanakarani P, et al. Primary Sjögren's Syndrome - Clinical and Immunological Features. J Ind Rheumatol Assoc 2003; 11: 63-5.

[16] Chisholm DM, Mason DK. Labial salivary gland biopsy in Sjögren's disease. J ClinPathol 1968; 21: 656-60.

[17] Everitt BS, Dunn G. Applied Multivariate Data Analysis. $2^{\text {nd }}$ ed. London: Arnold 2001.
[18] Johnson RA, Wichern DW. Applied Multivariate Statistical Analysis. $5^{\text {th }}$ ed. New Delhi: Pearson Education Asia 2002.

[19] Hosmer D, Lemeshow S. Applied Logistic Regression. $2^{\text {nd }}$ ed. New York: John Wiley \& Sons 2000.

[20] Priori R, Iannuccelli C, Alessandri C, et al. Fatigue in Sjögren's syndrome: relationship with fibromyalgia, clinical and biologic features. Clin Exp Rheumatol 2010; 28: S82-6.

[21] Segal B, Thomas W, Rogers T, et al. Prevalence, severity, and predictors of fatigue in subjects with primary Sjögren's syndrome. Arthritis Rheum 2008; 59: 1780-7.

[22] Aasarød K, Haga HJ, Berg KJ, et al. Renal involvement in primary Sjögren's syndrome. QJM 2000; 93: 297-304.

[23] Pertovaara M, Korpela M, Kouri T, Pasternack A. The occurrence of renal involvement in primary Sjögren's syndrome: a study of 78 patients. Rheumatology 1999; 38: 1113-20.

[24] Alexander E, Arnett F, Provost T. Sjögren's syndrome: association of anti-SSa antibodies with vasculitis, immunologic abnormalities and serologic hyperactivity. Ann Med Int 1983; 98: 155-9.

[25] Voulgarelis M, Ziakas PD, Papageorgiou A, Baimpa E, Tzioufas AG, Moutsopoulos HM. Prognosis and outcome of non-Hodgkin lymphoma in primary Sjögren syndrome. Medicine (Baltimore) 2012; 91: 1-9.

[26] Brito-Zeron P, Ramos-Casals M, Bove A, Sentis J, Font J. Predicting adverse outcomes in primary Sjögren's syndrome:identification of prognostic factors. Rheumatology 2007; 46: 1359-62.

[27] Seror R, Mariette X, Bowman S, et al. European League Against Rheumatism Sjögren's Task Force. Accurate detection of changes in disease activity in primary Sjögren's syndrome by the European League Against Rheumatism Sjögren's Syndrome Disease Activity Index. Arthritis Care Res 2010; 62: 551-8.

[28] Seror R, Ravaud P, Mariette X, et al. EULAR Sjögren's Task Force. EULAR Sjogren Syndrome Patient Reported Index (ESSPRI) development of a consensus patient index for primary Sjogren syndrome. Ann Rheum Dis 2011; 70: 968-72.

[29] Fox RI, Chan E, Benton L, Fong S, Friedlaender M, Howell FV. Treatment of primary Sjögren's syndrome with hydroxychloroquine. Am J Med 1988; 85: 62.

[30] Ramos-Casals M, Brito-Zerón P, Sisó-Almirall A, Bosch X, Tzioufas AG. Topical and systemic medications for the treatment of primary Sjögren's syndrome. Nat Rev Rheumatol 2012; 8: 399-411.

[31] Rihl M, Ulbricht K, Schmidt RE, Witte T. Treatment of sicca symptoms with hydroxychloroquine in patients with Sjögren's syndrome. Rheumatology 2009; 48: 796-9.

[32] Skopouli FN, Jagiello P, Tsifetaki N, Moutsopoulos HM. Methotrexate in primary Sjögren's syndrome. Clin Exp Rheumatol 1996; 14: 555.

[33] Willeke P, Schlüter B, Becker H, Schotte H, Domschke W, Gaubitz M. Mycophenolate sodium treatment in patients with primary Sjögren syndrome: a pilot trial. Arthritis Res Ther 2007; 9: R115.

[34] Endres S, Ghorbani R, Kelley VE, et al. The effect of dietary supplementation with n-3 polyunsaturated fatty acids on the synthesis of interleukin-1 and tumor necrosis factor by mononuclear cells. $\mathrm{N}$ Engl $\mathrm{J}$ Med 1989; 320: 265-71.

[35] Galarraga B, Ho M, Youssef HM, et al. Cod liver oil (n-3 fatty acids) as a non-steroidal anti-inflammatory drug sparing agent in rheumatoid arthritis. Rheumatology (Oxford) 2008; 47: 665-9.

[36] Fox RI, Stern M. Sjögren's syndrome: mechanisms of pathogenesis involve interaction of immune and neurosecretory systems. Scand J Rheumatol Suppl 2002; 116: 3-13. 